\title{
QUEEN'S
UNIVERSITY
BELFAST
}

\section{Sublinear Longest Path Transversals}

James A. Long, J., Milans, K. G., \& Munaro, A. (2021). Sublinear Longest Path Transversals. SIAM Journal on Discrete Mathematics, 35(3), 1673-1677. https://doi.org/10.1137/20M1362577

\section{Published in:}

SIAM Journal on Discrete Mathematics

\section{Document Version:}

Peer reviewed version

\section{Queen's University Belfast - Research Portal:}

Link to publication record in Queen's University Belfast Research Portal

\section{Publisher rights}

Copyright 2021Society for Industrial and Applied Mathematics. This work is made available online in accordance with the publisher's policies. Please refer to any applicable terms of use of the publisher.

\section{General rights}

Copyright for the publications made accessible via the Queen's University Belfast Research Portal is retained by the author(s) and / or other copyright owners and it is a condition of accessing these publications that users recognise and abide by the legal requirements associated with these rights.

Take down policy

The Research Portal is Queen's institutional repository that provides access to Queen's research output. Every effort has been made to ensure that content in the Research Portal does not infringe any person's rights, or applicable UK laws. If you discover content in the Research Portal that you believe breaches copyright or violates any law, please contact openaccess@qub.ac.uk. 


\title{
Sublinear Longest Path Transversals
}

\author{
James A. Long Jr. ${ }^{* 1}$, Kevin G. Milans ${ }^{\dagger 1}$, and Andrea Munaro ${ }^{\ddagger 2}$ \\ ${ }^{1}$ Department of Mathematics, West Virginia University, USA \\ ${ }^{2}$ School of Mathematics and Physics, Queen's University Belfast, UK
}

April 15, 2021

\begin{abstract}
We show that connected graphs admit sublinear longest path transversals. This improves an earlier result of Rautenbach and Sereni and is related to the fifty-year-old question of whether connected graphs admit longest path transversals of constant size. The same technique allows us to show that 2-connected graphs admit sublinear longest cycle transversals.
\end{abstract}

\section{Introduction}

A classical exercise in graph theory is to show that if $P$ and $Q$ are longest paths in a connected graph, then the vertex sets of $P$ and $Q$ have non-empty intersection (see [8], exercise 1.2.40). In 1966, Gallai [2] asked whether this result could be strengthened to assert that the family of all longest paths in a connected graph $G$ has non-empty intersection. It turns out the answer is no, as shown by Walther [6] with a 25-vertex counterexample. A 12-vertex counterexample, due to Walther and Voss [7] and independently Zamfirescu [10], is obtained from the Petersen graph by replacing one vertex $v$ with an independent set $\left\{v_{1}, v_{2}, v_{3}\right\}$ such that each $v_{i}$ becomes an endpoint of an edge incident to $v$ (see Figure 1).

Since Gallai's question has a negative answer, a single vertex is generally insufficient to meet every longest path in a connected graph $G$. A longest path transversal in $G$ is a set of vertices that intersects every longest path. Such a set is a transversal in the hypergraph on $V(G)$ whose edges are the vertex sets of longest paths in $G$. Let lpt $(G)$ be the minimum size of a longest path transversal in $G$. The graph $G_{0}$ in Figure 1 is a connected 12-vertex graph with $\operatorname{lpt}\left(G_{0}\right)=2$. Grünbaum [3] constructed a connected 324-vertex graph $G$ with $\operatorname{lpt}(G)=3$. Soon afterward, Zamfirescu [10] found such a graph with 270 vertices. Walther [6] and Zamfirescu [9] asked if $\operatorname{lpt}(G)$ is bounded for connected graphs $G$, and this remains

\footnotetext{
*jalong@mix.wvu.edu

†milans@math.wvu.edu

†a.munaro@qub.ac.uk
} 


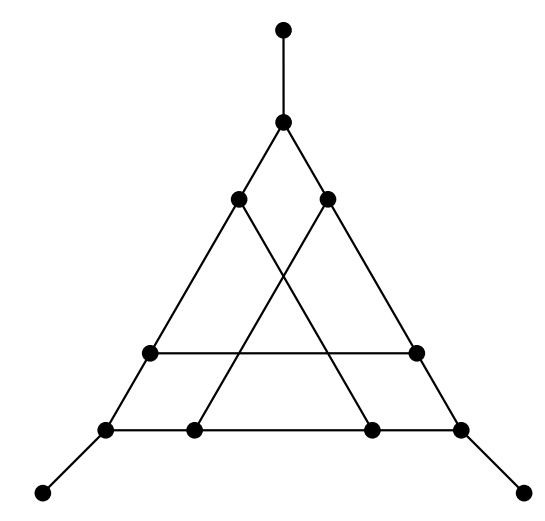

Figure 1: The graph $G_{0}$ : a 12 -vertex graph with $\operatorname{lpt}\left(G_{0}\right)=2$.

open. In fact, it is not known whether there is a connected graph $G$ with $\operatorname{lpt}(G) \geq 4$. Let $G$ be a connected graph. Since a connected graph does not contain vertex-disjoint longest paths, every partition of $V(G)$ into two sets has a part that contains no longest path in $G$, forcing the other part to be a longest path transversal. Applying this to a partition of $V(G)$ into two parts of nearly equal size gives $\operatorname{lpt}(G) \leq\lceil n / 2\rceil$ when $G$ is an $n$-vertex connected graph. It is not too difficult to improve this argument to obtain $\operatorname{lpt}(G) \leq\lceil n / 4\rceil$. Rautenbach and Sereni [4] showed that $\operatorname{lpt}(G) \leq\left\lceil\frac{n}{4}-\frac{n^{2 / 3}}{90}\right\rceil$ for every connected $n$-vertex graph $G$. We show that $\operatorname{lpt}(G) \leq 8 n^{3 / 4}$ when $G$ is an $n$-vertex connected graph, implying that connected graphs have sublinear longest path transversals.

Let $\operatorname{lct}(G)$ be the minimum size of a set of vertices $S$ such that $S$ intersects every longest cycle in $G$. Analogously to the case of longest paths in 1-connected graphs, every pair of longest cycles in a 2-connected graph intersect. The Petersen graph $G$ is 2-connected and $\operatorname{lct}(G)=2$. With no connectivity assumptions, Thomassen $[5]$ showed that $\operatorname{lct}(G) \leq\lceil n / 3\rceil$ for each $n$-vertex graph $G$. The bound is sharp when $G$ is a disjoint union of triangles and nearly sharp in the 1-connected case when $G$ is obtained from a star with $(n-1) / 3$ leaves by replacing each leaf with a triangle. On the other hand, Rautenbach and Sereni [4] proved that if $G$ is 2 -connected, then $\operatorname{lct}(G) \leq\left\lceil\frac{n}{3}-\frac{n^{2 / 3}}{36}\right\rceil$. We show that $\operatorname{lct}(G) \leq 20 n^{3 / 4}$ when $G$ is 2-connected (Corollary 2).

The problems of finding small longest path transversals and small longest cycle transversals are special cases of a general problem that we aim to address. Given a multigraph $F$ and an edge $e \in E(F)$ with endpoints $u$ and $v$, the subdivision operation produces a new multigraph $F^{\prime}$ in which $e$ is replaced by a path $u w v$ through a new vertex $w$ in $F^{\prime}$. A subdivision of $F$ is a graph obtained from $F$ via a sequence of zero or more subdivision operations. For a multigraph $R$ and a graph $G$, an $R$-subdivision in $G$ is a subgraph of $G$ isomorphic to a subdivision of $R$. We ask for a small set of vertices in $G$ that intersects every $R$-subdivision in $G$ of maximum size. The cases of longest path transversals and longest cycle transversals arise as $R=P_{2}$ and $R=C_{2}$ (the multigraph 2-vertex cycle), respectively. We prove that for each connected multigraph $R$, if the family $\mathcal{F}$ of maximum $R$-subdivisions in $G$ is pairwise intersecting, then $\mathcal{F}$ admits a transversal of size at most $C n^{3 / 4}$, where $C$ is a constant depending on $R$. 


\section{Maximum subdivision transversals}

Let $R$ be a multigraph. Recall that an $R$-subdivision in $G$ is a subgraph of $G$ isomorphic to a subdivision of $R$, and a maximum $R$-subdivision is an $R$-subdivision $F$ in $G$ that maximizes $|V(F)|$. An $R$-transversal of $G$ is a set of vertices intersecting each maximum $R$-subdivision. Let $\tau_{R}(G)$ be the minimum size of an $R$-transversal in $G$.

Given sets of vertices $X$ and $Y$ of $G$, an $(X, Y)$-separator is a set of vertices $S$ such that no path in $G-S$ has one endpoint in $X$ and the other endpoint in $Y$. We allow an $(X, Y)$-separator to contain vertices in $X$ and $Y$. An $(X, Y)$-connector is a collection of vertex-disjoint paths $\left\{P_{1}, \ldots, P_{k}\right\}$ such that each $P_{i}$ has one endpoint in $X$, the other endpoint in $Y$, and the interior vertices of $P_{i}$ are outside $X \cup Y$. A variant of Menger's Theorem asserts that the minimum size of an $(X, Y)$-separator equals the maximum size of an $(X, Y)$-connector (see, e.g., Theorem 3.3.1 in [1]).

Our next result shows that when the maximum $R$-subdivisions in a graph $G$ pairwise intersect, $G$ has sublinear $R$-transversals. We make no attempt to optimize the multiplicative constant 8 or the dependence on $m$.

Theorem 1. Let $R$ be a connected $m$-edge multigraph with $m \geq 1$ and let $G$ be an $n$-vertex graph. If the maximum $R$-subdivisions in $G$ pairwise intersect, then $\tau_{R}(G) \leq 8 m^{5 / 4} n^{3 / 4}$.

Proof. Let $m=|E(R)|$ and let $\varepsilon=2(m / n)^{1 / 4}$. We may assume that $m \leq n$, since otherwise we may take $V(G)$ as our $R$-transversal. Let $\mathcal{F}$ be the family of maximum $R$-subdivisions in $G$. An $\varepsilon$-partial transversal is a triple $(H, X, Y)$ such that $H$ is a subgraph of $G, X=V(G)-$ $V(H), Y \subseteq X$ with $|Y| \leq \varepsilon|X|$, and each $F \in \mathcal{F}$ is a subgraph of $H$ or contains a vertex in $Y$. Given an $\varepsilon$-partial transversal $(H, X, Y)$, we either obtain an $\varepsilon$-partial transversal $\left(H^{\prime}, X^{\prime}, Y^{\prime}\right)$ with $\left|V\left(H^{\prime}\right)\right|<|V(H)|$ or we produce an $R$-transversal with at most $8 m^{5 / 4} n^{3 / 4}$ vertices. Starting with $(H, X, Y)=(G, \varnothing, \varnothing)$ and iterating gives the result.

Let $(H, X, Y)$ be an $\varepsilon$-partial transversal, and let $\mathcal{F}_{0}$ be the set of $F \in \mathcal{F}$ such that $F$ is a subgraph of $H$. We may assume that $H$ contains vertex-disjoint paths $P_{1}$ and $P_{2}$ each of size $\lceil\varepsilon n\rceil$. Otherwise, every path in $H$ has size less than $2\lceil\varepsilon n\rceil$, and so each $F \in \mathcal{F}_{0}$ has at most $2 m\lceil\varepsilon n\rceil$ vertices. Since $\mathcal{F}_{0}$ is pairwise intersecting, we have that $V(F) \cup Y$ is an $R$-transversal for each $F \in \mathcal{F}_{0}$. It follows that $\tau_{R}(G) \leq|Y|+2 m\lceil\varepsilon n\rceil \leq \varepsilon n+2 m\lceil\varepsilon n\rceil \leq$ $(2 m+1) \varepsilon n+2 m \leq(2 m+2) \varepsilon n \leq 4 m \varepsilon n=8 m^{5 / 4} n^{3 / 4}$.

Suppose that $H$ has a $\left(V\left(P_{1}\right), V\left(P_{2}\right)\right)$-separator $S$ of size at most $\varepsilon^{2} n$. Since graphs in $\mathcal{F}_{0}$ are connected, each $F \in \mathcal{F}_{0}$ has a vertex in $S$ or is contained in some component of $H-S$. Also, since $\mathcal{F}_{0}$ is pairwise intersecting, at most one component $H^{\prime}$ of $H-S$ contains graphs in $\mathcal{F}_{0}$. Since $S$ is a separator, $H^{\prime}$ is disjoint from at least one of $\left\{P_{1}, P_{2}\right\}$. With $X^{\prime}=V(G)-V\left(H^{\prime}\right)$ and $Y^{\prime}=Y \cup S$, we have $\left|X^{\prime}\right|-|X| \geq \varepsilon n$ and $\left|Y^{\prime}\right|=|Y|+|S| \leq$ $\varepsilon|X|+\varepsilon^{2} n \leq \varepsilon|X|+\varepsilon\left(\left|X^{\prime}\right|-|X|\right) \leq \varepsilon\left|X^{\prime}\right|$. It follows that $\left(H^{\prime}, X^{\prime}, Y^{\prime}\right)$ is an $\varepsilon$-partial transversal. Also $\left|V\left(H^{\prime}\right)\right|<|V(H)|$ since $\left|X^{\prime}\right|>|X|$.

Otherwise, by Menger's Theorem, $H$ has a $\left(V\left(P_{1}\right), V\left(P_{2}\right)\right)$-connector $\mathcal{P}$ with $|\mathcal{P}| \geq \varepsilon^{2} n$. Let $\mathcal{P}^{\prime}$ be the set of paths in $\mathcal{P}$ of size at most $2 / \varepsilon^{2}$. Note that $\left|\mathcal{P}^{\prime}\right| \geq|\mathcal{P}| / 2$, or else $\mathcal{P}$ has at least $\left(\varepsilon^{2} n\right) / 2$ paths of size more than $2 / \varepsilon^{2}$, contradicting that the paths in $\mathcal{P}$ are disjoint. So we have $\left|\mathcal{P}^{\prime}\right| \geq|\mathcal{P}| / 2 \geq\left(\varepsilon^{2} / 2\right) n=2 m^{1 / 2} n^{1 / 2} \geq 2$. Combining $P_{1}$ with two paths in $\mathcal{P}^{\prime}$ whose endpoints in $V\left(P_{1}\right)$ are as far apart as possible and a segment of $P_{2}$ gives a cycle $C_{0}$ such that $\left(\varepsilon^{2} / 2\right) n \leq\left|V\left(C_{0}\right)\right| \leq 2\lceil\varepsilon n\rceil+4 / \varepsilon^{2}-4 \leq 2 \varepsilon n+4 / \varepsilon^{2}$, where the lower bound 


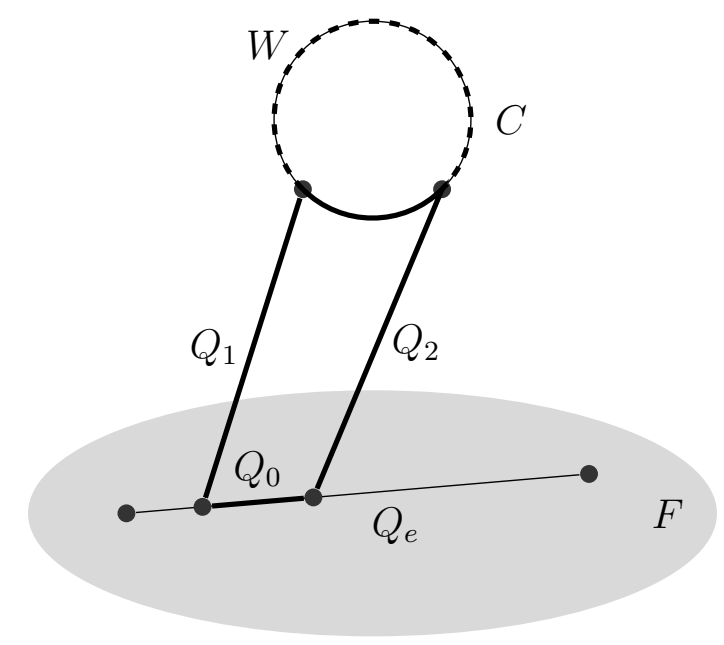

Figure 2: $(V(C), V(F))$-connector case. The subpath $W$ of the cycle $C$ is dashed, and the cycle $D$ is displayed in bold.

counts vertices in $V\left(P_{1}\right) \cap V\left(C_{0}\right)$ and the upper bound counts at most $2\lceil\varepsilon n\rceil$ vertices in $\left(V\left(P_{1}\right) \cup V\left(P_{2}\right)\right) \cap V\left(C_{0}\right)$, at most $4 / \varepsilon^{2}$ vertices on the paths in $\mathcal{P}^{\prime}$ linking $P_{1}$ and $P_{2}$, and observing that the 4 endpoints of the linking paths are counted twice.

Let $C$ be a longest cycle in $H$ subject to $|V(C)| \leq 2 \varepsilon n+4 / \varepsilon^{2}$, let $\ell=|V(C)|$, and note that $\ell \geq\left|V\left(C_{0}\right)\right| \geq\left(\varepsilon^{2} / 2\right) n$. If $V(C)$ intersects each subgraph in $\mathcal{F}_{0}$, then $Y \cup V(C)$ witnesses $\tau_{R}(G) \leq|V(C)|+|Y| \leq\left(2 \varepsilon n+4 / \varepsilon^{2}\right)+\varepsilon n=3 \varepsilon n+(n / m)^{1 / 2}<8 m^{5 / 4} n^{3 / 4}$. Otherwise, choose $F \in \mathcal{F}_{0}$ that is disjoint from $C$. We may assume $|V(F)| \geq \ell$, or else $Y \cup V(F)$ witnesses that $\tau_{R}(G) \leq|V(F)|+|Y|<\left(2 \varepsilon n+4 / \varepsilon^{2}\right)+\varepsilon n<8 m^{5 / 4} n^{3 / 4}$.

If $H$ has a $(V(C), V(F))$-separator $T$ of size at most $\varepsilon \ell$, then we obtain an $\varepsilon$-partial transversal as follows. At most one component $H^{\prime}$ of $H-T$ contains graphs in $\mathcal{F}_{0}$. Let $X^{\prime}=V(G)-V\left(H^{\prime}\right)$ and let $Y^{\prime}=Y \cup T$. Since $H^{\prime}$ is disjoint from one of $\{C, F\}$, it follows that $\left|X^{\prime}\right|-|X| \geq \ell$. We compute $\left|Y^{\prime}\right|=|Y|+|T| \leq \varepsilon|X|+\varepsilon \ell \leq \varepsilon|X|+\varepsilon\left(\left|X^{\prime}\right|-|X|\right) \leq \varepsilon\left|X^{\prime}\right|$. Hence $\left(H^{\prime}, X^{\prime}, Y^{\prime}\right)$ is an $\varepsilon$-partial transversal with $\left|V\left(H^{\prime}\right)\right|<|V(H)|$.

Otherwise, $H$ has a $(V(C), V(F))$-connector $\mathcal{Q}$ with $|\mathcal{Q}| \geq \varepsilon \ell$. We use $\mathcal{Q}$ to obtain a contradiction. For $e \in E(R)$, let $Q_{e}$ be the path in $F$ corresponding to $e$, and let $\mathcal{Q}_{e}$ be the set of paths in $\mathcal{Q}$ which have an endpoint in $Q_{e}$. Since $|E(R)|=m$, it follows that $\left|\mathcal{Q}_{e}\right| \geq|\mathcal{Q}| / m \geq \varepsilon \ell / m$ for some edge $e \in E(R)$. Let $\mathcal{Q}^{\prime}$ be the set of paths in $\mathcal{Q}_{e}$ of size at most $\frac{2 m n}{\varepsilon \ell}$, and note that $\left|\mathcal{Q}^{\prime}\right| \geq\left|\mathcal{Q}_{e}\right| / 2 \geq \frac{\varepsilon \ell}{2 m}$, or else $\mathcal{Q}_{e}$ has at least $\frac{\varepsilon \ell}{2 m}$ paths of size more than $\frac{2 m n}{\varepsilon \ell}$, a contradiction. The endpoints of paths in $\mathcal{Q}^{\prime}$ divide $Q_{e}$ into $\left|\mathcal{Q}^{\prime}\right|-1$ edge-disjoint subpaths. Choose $Q_{1}, Q_{2} \in \mathcal{Q}^{\prime}$ to minimize the length of such a subpath $Q_{0}$ of $Q_{e}$, and note that $Q_{0}$ has length at most $\frac{n-1}{\left|\mathcal{Q}^{\prime}\right|-1}$; see Figure 2 . Since $m \leq n$, we have $2 m \leq 2 m^{3 / 4} n^{1 / 4}=\frac{\varepsilon^{3}}{4} n \leq \frac{\varepsilon \ell}{2}$, and hence $\frac{n-1}{\left|\mathcal{Q}^{\prime}\right|-1}<\frac{n}{\frac{\varepsilon \ell}{2 m}-1}=\frac{2 m n}{\varepsilon \ell-2 m} \leq \frac{4 m n}{\varepsilon \ell}$.

The endpoints of $Q_{1}$ and $Q_{2}$ on $C$ partition $C$ into two subpaths; let $W$ be the longer subpath. If $|E(W)| \geq\left|E\left(Q_{0}\right)\right|$, then we would obtain a larger $R$-subdivision by using $Q_{1}$, $W$, and $Q_{2}$ to bypass $Q_{0}$. Since $F$ is a maximum $R$-subdivision, we have $|E(W)|<\left|E\left(Q_{0}\right)\right|$. Therefore using $Q_{1}, Q_{0}$, and $Q_{2}$ to bypass $W$ gives a cycle $D$ with $|E(D)|>|E(C)|$. By the extremal choice of $C$, it follows that $|V(D)|>2 \varepsilon n+4 / \varepsilon^{2}$. On the other hand, $|V(D)|=$ 
$|E(D)| \leq \frac{\ell}{2}+\left|E\left(Q_{1}\right)\right|+\left|E\left(Q_{0}\right)\right|+\left|E\left(Q_{2}\right)\right| \leq \frac{\ell}{2}+\frac{2 m n}{\varepsilon \ell}+\frac{4 m n}{\varepsilon \ell}+\frac{2 m n}{\varepsilon \ell}=\frac{\ell}{2}+\frac{8 m n}{\varepsilon \ell}$.

Therefore $2 \varepsilon n+\frac{4}{\varepsilon^{2}}<|V(D)| \leq \frac{\ell}{2}+\frac{8 m n}{\varepsilon \ell} \leq \varepsilon n+\frac{2}{\varepsilon^{2}}+\frac{8 m n}{\varepsilon \ell} \leq \varepsilon n+\frac{2}{\varepsilon^{2}}+\frac{16 m}{\varepsilon^{3}}$, where the last inequality uses $\ell \geq\left(\varepsilon^{2} / 2\right) n$. Simplifying gives $\varepsilon n<\frac{16 m}{\varepsilon^{3}}-\frac{2}{\varepsilon^{2}}<\frac{16 m}{\varepsilon^{3}}$, and this inequality is violated when $\varepsilon \geq(16 \mathrm{~m} / \mathrm{n})^{1 / 4}$.

Applying Theorem 1, we obtain the following corollary.

Corollary 2. Let $G$ be an $n$-vertex graph. If $G$ is connected, then $\operatorname{lpt}(G) \leq 8 n^{3 / 4}$. If $G$ is 2-connected, then $\operatorname{lct}(G) \leq 20 n^{3 / 4}$.

Proof. When $R=P_{2}$, an $R$-transversal is a longest path transversal. It is well known that if $G$ is connected, then the longest paths pairwise intersect. By Theorem 1, we have $\operatorname{lpt}(G)=\tau_{R}(G) \leq 8 n^{3 / 4}$.

Similarly, when $R=C_{2}$, an $R$-transversal is a longest cycle transversal. If $G$ is 2 connected, then the longest cycles pairwise intersect. By Theorem 1, we have $\operatorname{lct}(G)=$ $\tau_{R}(G) \leq 8 \cdot 2^{5 / 4} \cdot n^{3 / 4} \leq 20 n^{3 / 4}$.

We do not know whether the assumption in Theorem 1 that $R$ is connected is necessary to obtain sublinear $R$-transversals. To obtain analogues of Corollary 2 for general $R$, we show that the maximum $R$-subdivisions pairwise intersect when the connectivity of $G$ is sufficiently large. Recall that a graph $G$ is $k$-connected if $|V(G)|>k$ and $G-S$ is connected for each $S \subseteq V(G)$ with $|S|<k$. Moreover, the connectivity of $G$, denoted $\kappa(G)$, is the maximum $k$ such that $\mathrm{G}$ is $k$-connected.

Lemma 3. Let $R$ be a connected $m$-edge multigraph with $m \geq 1$. If $\kappa(G)>m^{2}$, then the maximum $R$-subdivisions in $G$ are pairwise intersecting.

Proof. Suppose for a contradiction that $G$ has disjoint maximum $R$-subdivisions $F_{1}$ and $F_{2}$, and let $k=\left|V\left(F_{1}\right)\right|=\left|V\left(F_{2}\right)\right|$. By Menger's Theorem, there is an $\left(V\left(F_{1}\right), V\left(F_{2}\right)\right)$-connector $\mathcal{P}$ with $|\mathcal{P}|=\min \left\{k, m^{2}+1\right\}$. If $|\mathcal{P}|=k$, then every vertex in $F_{1}$ is an endpoint of a path in $\mathcal{P}$, and we obtain an $R$-subdivision of size more than $k$ by replacing an edge $u v \in E\left(F_{1}\right)$ with a path in $\mathcal{P}$ having $u$ as an endpoint, a path in $\mathcal{P}$ having $v$ as an endpoint, and an appropriate path in the connected subgraph $F_{2}$.

So we may assume $|\mathcal{P}|=m^{2}+1$. For each $e \in E(R)$, let $F_{i}(e)$ be the path in $F_{i}$ corresponding to $e$. Since $R$ has no isolated vertices, we may associate each $P \in \mathcal{P}$ with an ordered pair of edges $\left(e_{1}, e_{2}\right) \in(E(R))^{2}$ such that $P$ has its endpoint in $F_{1}$ in $F_{1}\left(e_{1}\right)$ and its endpoint in $F_{2}$ in $F_{2}\left(e_{2}\right)$. Since $|\mathcal{P}|>m^{2}$, some pair $\left(e_{1}, e_{2}\right)$ is associated with distinct paths $P, Q \in \mathcal{P}$. Let $W_{i}$ be the subpath of $F_{i}\left(e_{i}\right)$ whose endpoints are in $V(P) \cup V(Q)$. If $\left|E\left(W_{1}\right)\right| \geq\left|E\left(W_{2}\right)\right|$, then we modify $F_{2}$ to obtain a larger $R$-subdivision by using $P, W_{1}$, and $Q$ to bypass $W_{2}$. Similarly, if $\left|E\left(W_{2}\right)\right| \geq\left|E\left(W_{1}\right)\right|$, then we modify $F_{1}$ to obtain a larger $R$-subdivision by using $P, W_{2}$, and $Q$ to bypass $W_{1}$.

Corollary 4. Let $R$ be a connected m-edge multigraph. If $G$ is an n-vertex graph with $\kappa(G)>m^{2}$, then $\tau_{R}(G) \leq 8 m^{5 / 4} n^{3 / 4}$.

As it is not known whether there exists a connected graph $G$ with $\operatorname{lpt}(G)>3$, reducing the gap between our sublinear upper bound on $\operatorname{lpt}(G)$ and the constant lower bound remains a major open problem in the area of longest path transversals. 


\section{Acknowledgement}

The authors greatly appreciate the careful comments of an anonymous referee.

\section{References}

[1] R. Diestel. Graph Theory. Graduate Texts in Mathematics. Springer, 2005.

[2] T. Gallai. Problem 4. In P. Erdős and G. Katona, editors, Theory of Graphs, Proceedings of the Colloquium Held at Tihany, Hungary, September 1966, page 362. Academic Press, New York, 1968.

[3] B. Grünbaum. Vertices missed by longest paths or circuits. Journal of Combinatorial Theory, Series A, 17(1):31-38, 1974.

[4] D. Rautenbach and J.-S. Sereni. Transversals of longest paths and cycles. SIAM Journal on Discrete Mathematics, 28(1):335-341, 2014.

[5] C. Thomassen. Hypohamiltonian graphs and digraphs. In Y. Alavi and D.R. Lick, editors, Theory and Applications of Graphs, pages 557-571. Springer Berlin Heidelberg, 1978.

[6] H. Walther. Über die nichtexistenz eines knotenpunktes, durch den alle längsten wege eines graphen gehen. Journal of Combinatorial Theory, 6(1):1-6, 1969.

[7] H. Walther and H.-J. Voss. Über Kreise in Graphen. Deutscher Verlag der Wissenschaften, 1974.

[8] D. B. West. Introduction to Graph Theory. Prentice Hall, 2nd edition, 2001.

[9] T. Zamfirescu. A two-connected planar graph without concurrent longest paths. Journal of Combinatorial Theory, Series B, 13(2):116-121, 1972.

[10] T. Zamfirescu. On longest paths and circuits in graphs. Mathematica Scandinavica, 38: 211-239, 1976. 